\title{
Effects of Aerobic Exercise Training in Community-Based Subjects Aged 80 and Older: A Pilot Study
}

\author{
Peter V. Vaitkevicius, MD, * Caroline Ebersold, PA, ${ }^{\dagger}$ Muhammad S. Shah, BA, * \\ Nikita S. Gill, MD, ${ }^{+}$Robert L. Katz, BA, Matthew J. Narrett, MD, ${ }^{\ddagger}$ Gary E. Applebaum, MD, $\neq$ \\ Sheryl M. Parrish, MSW, ACSW, LSCW-C, ${ }^{\ddagger}$ Frances C. O’Connor, ${ }^{\dagger}$ and Jerome L. Fleg, MD ${ }^{\dagger}$
}

OBJECTIVES: To assess the ability of sedentary, frail subjects aged 80 and older to train in a community-based exercise program and to evaluate clinical factors that predict improvements in peak oxygen consumption $\left(\mathrm{VO}_{2}\right.$ peak $)$.

DESIGN: Pretest, posttest.

SETTING: Charlestown Retirement Community, Catonsville, Maryland

PARTICIPANTS: Twenty-two (11 male, 11 female; mean age \pm standard deviation $=84 \pm 4.0$, range $80-92$ ) selfreferred.

INTERVENTION: Six months of moderate-intensity aerobic exercise training, two to three sessions/week, 20 to 30 minutes per session. Training modes included treadmill walking and/or stationary cycling.

MEASUREMENTS: Baseline and follow-up maximal exercise treadmill tests (ETTs) with electrocardiogram monitoring and respiratory gas analysis.

RESULTS: Six months of aerobic exercise training resulted in significant increases (mean \pm standard deviation) in ETT duration $(11.9 \pm 3.3$ vs $15.9 \pm 4.3$ minutes; $P=$ $.01), \mathrm{VO}_{2}$ peak $(1.23 \pm 0.37$ vs $1.31 \pm 0.36 \mathrm{~L} / \mathrm{min} ; P=$ $.04)$, and oxygen pulse $(9.3 \pm 2.8$ vs $10.1 \pm 3.2 ; P=.03)$.

From the "Division of Geriatrics and Cardiology, University of Michigan Medical School and Veterans Affairs Ann Arbor Healthcare System/Geriatric Research Education and Clinical Center (11G), Ann Arbor, Michigan; ${ }^{+}$Laboratory of Cardiovascular Science, Gerontology Research Center, National Institute of Aging, National Institutes of Health, Baltimore, Mary-

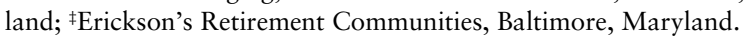

This study was supported in part by the Brookdale National Fellowship Program; the Department of Veterans Affairs Ann Arbor Geriatric Research Education and Clinical Center; the John A. Hartford Foundation Grant "Strengthening Training in Geriatrics for Physicians" (97215-G); the Johns Hopkins Teaching Nursing Home Award (PO-1-AG-04402); the National Institute of Aging, National Institutes of Health; and Quinton Instruments Co., Bothell, Washington.

Address correspondence to Peter V. Vaitkevicius, MD, VA Ann Arbor Healthcare System/GRECC (11G), 2215 Fuller Road, Ann Arbor, MI 48105. E-mail: pvait@umich.edu
Mean heart rate was significantly lower during submaximal ETT stages 1 through $4(P<.05)$, and resting systolic blood pressure decreased $(146 \pm 18$ vs $133 \pm 14 \mathrm{mmHg}$; $P=.01)$ after training. Multiple regression analysis indicated that baseline $\mathrm{VO}_{2}$ peak $(r=0.75, P=.002)$ and the total amount of time spent in exercise training $(r=0.55$, $P=.008)$ were independent predictors of the trainingrelated improvements in $\mathrm{VO}_{2}$ peak.

CONCLUSION: Subjects aged 80 and older can increase aerobic capacity and reduce systolic blood pressure in a community-based exercise program of moderate intensity. The most important predictors of change in $\mathrm{VO}_{2}$ peak were baseline $\mathrm{VO}_{2}$ peak and the time spent in exercise training. Subjects with a lower baseline $\mathrm{VO}_{2}$ peak had the greatest improvements in $\mathrm{VO}_{2}$ peak after training. J Am Geriatr Soc 50:2009-2013, 2002.

Key words: aerobic training; older people; exercise; oxygen consumption

A $\mathrm{n}$ age-related reduction in maximal oxygen consumption $\left(\mathrm{VO}_{2} \mathrm{max}\right)$ has been well documented in both men and women. ${ }^{1-4}$ Between the ages of 30 and 70, $\mathrm{VO}_{2} \max$ has been shown to decline $9.1 \%$ per decade in active men and $7.5 \%$ in active women. ${ }^{3}$ By the seventh decade, this fall in aerobic capacity, along with a decline in muscle mass (30-40\%), ${ }^{5}$ strength $(30-40 \%),{ }^{6}$ and flexibility $(20-30 \%),{ }^{7}$ results in a decline in functional capacity. Furthermore, these losses have been shown to be more pronounced in the eighth decade and beyond, resulting in difficulty performing activities of daily living (ADLs). ${ }^{8-10}$ In a study by Morey et al., physical inactivity was shown to be an independent risk factor for functional decline. ${ }^{10}$ As the number of individuals reaching the age of 80 grows rapidly, maintaining functional capacity becomes a potential means of preventing or delaying institutionalization. . $^{5,8,11}$

Fortunately, age-associated declines in $\mathrm{VO}_{2} \max$ have been shown to be modifiable with regular physical activity. Endurance exercise training in older people results in 
improvements in functional capacity, as measured by increases in $\mathrm{VO}_{2}$ max. ${ }^{8,12}$ Clinical studies have shown that individuals aged 65 to 79 can benefit significantly from exercise training, improving their $\mathrm{VO}_{2} \max$ by $8 \%$ to $30 \%{ }^{11,13-17}$ Green et al. speculate that even a small improvement in $\mathrm{VO}_{2}$ max, can mean the difference between independent or assisted living. ${ }^{18,19}$

To our knowledge, there has been little research examining whether subjects aged 80 and older can benefit from aerobic exercise training. Given the exponential increase in disability beyond this age, whether functional capacity can be improved with training in the oldest old has particular relevance. Therefore, the purpose of this study was to investigate the effects of a practical communitybased aerobic exercise training program on peak treadmill oxygen consumption in subjects aged 80 and older.

\section{METHODS}

\section{Population}

Sedentary men and women aged 80 and older living in a community-based continuing care community (Charlestown Retirement Community, Catonsville, $\mathrm{MD}$ ) were recruited to participate in a 6-month aerobic exercise training program with baseline and follow-up maximal exercise treadmill testing (ETT). The community consists of 2,384 individuals ( $74 \%$ women, $26 \%$ men), $72 \%$ aged 80 and older, living independently $(83.6 \%)$, in assisted living facilities $(5.4 \%)$, and in convalescent care facilities $(11 \%)$ on a single campus. A training facility was opened on the campus in a centrally located facility within a short walking distance of subjects' apartments, as part of a health promotion program sponsored by the campus medical center and community administration. A full-time fitness instructor was on site to assist residents with their training. The majority of subjects moved to this continuing-care community secondary to current or impending medical illnesses, frailty, or the dependency of a spouse. Subjects were recruited using the community's newspapers and media services. The Johns Hopkins Bayview Medical Center Institutional Review Board approved the study before recruitment.

\section{Subject Recruitment Criteria}

Subjects were screened via an extensive medical history questionnaire in addition to the baseline physician-reviewed ETT. All subjects gave written informed consent and received clearance to participate in the exercise program from their primary care physician. To obtain a representative cohort of individuals in this age range, subjects with cardiovascular, pulmonary, neurological, or musculoskeletal diseases or conditions were included in the investigation. To avoid potential training-induced precipitation or exacerbation of myocardial ischemia, subjects demonstrating 2 $\mathrm{mm}$ or more of ST segment depression on the baseline treadmill test electrocardiogram (ECG) were excluded. Additional exclusion criteria included failure to attain a maximal effort during the baseline or follow-up treadmill test. A maximal ETT was defined by the achievement of a peak respiratory exchange ratio (RER) of 1.0 or greater and a peak heart rate (HR) of $85 \%$ or greater of the agepredicted value. Subjects were also excluded if they failed to attend at least $25 \%$ of the recommended three exercise sessions per week. Individuals who underwent the addition of beta-blocker therapy or the addition, removal, or change in dose of baseline cardiovascular medications during the investigation were excluded from the analysis.

\section{Baseline and Follow-up Testing}

Blood pressure measurements were taken in a seated position after 10 minutes of rest, using an analog sphygmomanometer on the right arm on the morning of testing, before any other measurements. Subjects then completed a survey assessing their ability to perform specific ADLs such as bathing, dressing, and walking (total of eight items) and instrumental activities of daily living (IADLs) such as shopping, meal preparation, and housework (total of seven items). ${ }^{20}$ Each activity was rated on a scale from 1 to 10 , representing the ease with which the activities were regularly performed..$^{21,22}$ Baseline and 6-month follow-up exercise testing was conducted according to a modified Balke protocol. Initial walking speeds varied among subjects (range 1.6-4.0 km/h) depending on individual ability. For each subject, the identical protocol was used during baseline and follow-up testing. Expired oxygen $\left(\mathrm{O}_{2}\right)$ and carbon dioxide concentrations were monitored continuously throughout exercise using Quinton Instrument QM3 Metabolic Cart (Bothell, WA) with 30-second averaging. The following variables were measured or derived from each ETT: (1) oxygen consumption $\left(\mathrm{VO}_{2}\right.$ peak) and RER, measured directly; (2) HR, monitored continuously via ECG; (3) blood pressure, obtained with a sphygmomanometer; (4) $\mathrm{O}_{2}$ pulse, defined as $\mathrm{VO}_{2}$ peak/HR; and (5) exercise test duration in minutes. A cardiologist, exercise physiologist, and an exercise technician were present for each testing session.

\section{Exercise Training Program}

For the first three to five sessions of the exercise program, each subject met with a fitness instructor for familiarization with the equipment. Subjects were encouraged to participate in treadmill walking (Quinton Medical Track CR60, Bothell, WA) and stationary cycling (Schwinn Airdyne, Boulder, CO) for three sessions per week for 20 to 30 minutes per session. After the supervised training sessions, subjects continued to train on their own with minimal input from the trainer. The instructor would facilitate the gradual increase in the workloads as directed by the subject and would provide casual supervision to ensure the safe operation of the training equipment. Before each session, subjects participated in 5 to 10 minutes of warm-up (treadmill walking at $1-1.6 \mathrm{~km} / \mathrm{h}$ ) and stretching exercises performed while standing (arm, trunk, and extremity flexion and extension) and, after each session, 5 minutes of cool-down exercises (treadmill walking at $1-1.6 \mathrm{~km} / \mathrm{h}$ ). Exercise intensity was prescribed at $60 \%$ to $80 \%$ of maximal HR, derived from the initial ETT. Using HR monitors (Cateye Co. Limited, Osaka, Japan), subjects were taught to monitor their HR response, and work rates were adjusted monthly to stay within prescribed HR ranges. Exercise intensity and frequency were moderate to mitigate the risk of injury in this frail population. Each subject recorded the average HR maintained during each training session in addition to the treadmill speed and grade, pedal 
revolutions per minute of the stationary cycle, and duration of exercise. Subjects were monitored continuously throughout their training sessions by a fitness instructor and encouraged to maintain their target HR throughout each session. Strength training was not promoted for this cohort. We attempted to maximize adherence to exercise by reinforcing their success at each session and promoting the benefits of exercise using the media services within the community (closed circuit television and newsletters). The training staff contacted subjects who participated less than twice per week for more than 2 consecutive weeks, to promote greater adherence. Participation in the exercise program was not dependent on rigorous compliance. It was the goal of the study to have the subjects consider the training program a permanent part of the community environment. If a subject wished to train less than the amount encouraged by the training staff, it was permitted. Thus, individuals were not threatened with specific compliance requirements, but those attending fewer than $25 \%$ of the scheduled exercise sessions were excluded from the data analysis.

\section{Statistical Analysis}

Exercise variables pre- and posttraining were compared using paired $t$ test analysis. Clinical correlates of traininginduced changes in exercise capacity were examined with simple linear regression. The independent effects of these variables on the change in $\mathrm{VO}_{2}$ peak were assessed in a stepwise multiple regression model. Only those variables that met $P<.10$ on univariate analysis were included in the stepwise analysis. The effect of aerobic training on $\mathrm{HR}, \mathrm{VO}_{2}$, and $\mathrm{O}_{2}$ pulse across submaximal work rates was assessed using a two-way repeated measures analysis of variance. Variables are expressed as mean \pm standard deviation. For all analyses, a two-tailed $P$-value $<.05$ was required for significance.

\section{RESULTS}

Thirty-five individuals (20 men, 15 women) volunteered for the training. Three subjects had prior coronary artery bypass graft surgery and nine had a history of hypertension. No subjects met ECG exclusion criteria for myocardial ischemia on the baseline ETT, and all were asymptomatic at the time of the investigation. Seven of the original 35 volunteers were excluded from the investigation because of an inability to reach an RER of 1.0 or greater during the baseline $(\mathrm{n}=5)$ or follow-up $(\mathrm{n}=2)$ ETT. During the training period, two subjects were excluded because of prolonged illness and three for attending fewer than $25 \%$ of the exercise sessions. Finally, one subject was eliminated because of the addition of beta-blocker therapy during the training program, leaving 22 subjects $(63 \%)$ (mean age $84 \pm 4 ; 11$ men, 11 women) who completed 6 months of exercise training with maximal baseline and follow-up ETTs. Of these 22 subjects, six $(27 \%)$ had a history of coronary artery disease, $10(43 \%)$ had arthritis, three $(14 \%)$ had a history of cancer, and two $(9 \%)$ had chronic atrial fibrillation. Cardiovascular medications included beta-blockers, two (9\%); calcium channel blockers, six (27\%); diuretics, $2(9 \%)$; digitalis, two $(9 \%)$; and anticoagulants, three $(14 \%)$.

The subjects exercised an average of $2.0 \pm 0.6$ days per week for $21 \pm 4$ minutes per session (treadmill min/ session, $11.4 \pm 4.0$ : bike min/session, $9.3 \pm 2.9$ ). The average training intensity was $76 \pm 8 \%$ of the maximal HR on the stationary bicycle and $75 \pm 8 \%$ of that on the treadmill. This intensity and volume of exercise resulted in no significant changes in weight or resting HR, but resting systolic blood pressure was significantly decreased after the training period (Table 1).

Table 1 describes variables measured during the ETT before and after the training period. Six months of exercise training resulted in a statistically significant $33 \%$ increase in the mean ETT duration. $\mathrm{VO}_{2}$ peak and $\mathrm{O}_{2}$ pulse at ex-

Table 1. Resting and Exercise Variables Pre- and Postaerobic Training

\begin{tabular}{|c|c|c|c|}
\hline \multirow[b]{2}{*}{ Variable } & Pre & Post & \multirow[b]{2}{*}{$P$-value* } \\
\hline & \multicolumn{2}{|c|}{ Mean \pm Standard Deviation } & \\
\hline \multicolumn{4}{|l|}{ Resting } \\
\hline Weight, kg & $67.4 \pm 13$ & $67.0 \pm 13$ & .5 \\
\hline Body mass index, $\mathrm{kg} / \mathrm{m}^{2}$ & $24.2 \pm 3.0$ & $24.0 \pm 3.0$ & .47 \\
\hline Heart rate, bpm & $76 \pm 13$ & $78 \pm 13$ & .38 \\
\hline $\mathrm{SBP}, \mathrm{mmHg}$ & $146 \pm 18$ & $133 \pm 4$ & .01 \\
\hline $\mathrm{DBP}, \mathrm{mmHg}$ & $78 \pm 25$ & $70 \pm 10$ & .15 \\
\hline \multicolumn{4}{|l|}{ Exercise } \\
\hline Duration, min & $11.9 \pm 3.3$ & $15.9 \pm 4.3$ & .01 \\
\hline $\mathrm{VO}_{2}$ peak, L/min & $1.23 \pm 0.37$ & $1.31 \pm 0.36$ & .04 \\
\hline $\mathrm{VO}_{2}$ peak, $\mathrm{L} / \mathrm{min} / \mathrm{kg}$ & $18.3 \pm 3.9$ & $19.4 \pm 3.5$ & .06 \\
\hline Peak heart rate, bpm & $133 \pm 16$ & $131 \pm 21$ & .62 \\
\hline Peak oxygen pulse & $9.3 \pm 2.8$ & $10.1 \pm 3.2$ & .03 \\
\hline Peak respiratory exchange ratio & $1.08 \pm 0.04$ & $1.08 \pm 0.05$ & .65 \\
\hline Peak SBP, mmHg & $190 \pm 16$ & $184 \pm 24$ & .22 \\
\hline Peak DBP, $\mathrm{mmHg}$ & $74 \pm 12$ & $73 \pm 12$ & .73 \\
\hline
\end{tabular}

${ }^{*}$ Paired $\mathrm{t}$ test analysis.

$\mathrm{SBP}=$ systolic blood pressure; $\mathrm{DBP}=$ diastolic blood pressure $; \mathrm{VO}_{2}$ peak $=$ peak oxygen consumption use 
Table 2. Univariate Correlates of Training-Induced Change in Peak Volume of Oxygen Use $\left(\mathrm{VO}_{2}\right.$ peak $)$

\begin{tabular}{lrr}
\hline \multicolumn{1}{c}{ Variable } & $\mathrm{r}$ & $P$-value \\
\hline Age & 0.33 & .14 \\
Baseline VO, peak, $\mathrm{mL} / \mathrm{min} / \mathrm{kg}$ & -0.47 & .03 \\
Total minutes of aerobic training & 0.55 & .01 \\
Number of aerobic training sessions & 0.51 & .02 \\
Number of aerobic training sessions per week & 0.42 & .01 \\
Minutes of aerobic training per session & 0.39 & .18 \\
Total minutes of bike exercise & 0.52 & .01 \\
Total minutes of treadmill exercise & 0.42 \\
\hline
\end{tabular}

haustion both increased significantly, by $6.5 \%$ and $8.6 \%$, respectively, but maximal HR, RER, systolic blood pressure, and diastolic blood pressure did not change with exercise training. In addition, analysis of variance (ANOVA) with repeated measures was used to determine whether HR, $\mathrm{VO}_{2}$, or $\mathrm{O}_{2}$ pulse were significantly different during the first four submaximal stages of the ETT pre- and posttraining. No significant differences were observed in $\mathrm{VO}_{2}$ or $\mathrm{O}_{2}$ pulse at any exercise stage, but HR was significantly lower, by an average of 10 beats per minute, at the followup ETT for stages 1 through 4, consistent with a training effect $(P<.05$, ANOVA with repeated measures, Bonferroni, post hoc analysis). Both sexes responded similarly to training for all variables examined.

To determine those variables that were predictive of a training-induced improvement in $\mathrm{VO}_{2}$ peak in this older cohort, the change in $\mathrm{VO}_{2}$ peak was examined as a function of age, baseline $\mathrm{VO}_{2}$ peak, the total time spent training (bicycle + treadmill exercise), the time spent in bicycle exercise alone, the time spent in treadmill exercise alone, and the total number of exercise sessions using linear regression analysis (Table 2). Significant positive correlations were demonstrated between the change in $\mathrm{VO}_{2}$ peak and the total exercise time, the total number of exercise sessions, and the time spent riding the stationary bicycle. A significant negative correlation was demonstrated between baseline $\mathrm{VO}_{2}$ peak and the change in $\mathrm{VO}_{2}$ peak after exercise training.

Multiple step-wise regression analysis was used to determine which of these variables were independent predictors of the training-induced change in $\mathrm{VO}_{2}$ peak (Table 3). Based on the univariate results, baseline $\mathrm{VO}_{2}$ peak, total exercise time, and the number of exercise sessions were included. In this model, time exercising (total aerobic minutes) and baseline $\mathrm{VO}_{2}$ peak $(\mathrm{mL} / \mathrm{min} / \mathrm{kg})$ were independent predictors of the increase in $\mathrm{VO}_{2}$ peak with exercise training (adjusted model $r^{2}=0.52$ ). Additionally, a history of coronary artery disease, hypertension, pulmonary disease, diabetes mellitus, neurologic disease, or arthritis was not an independent predictor of adherence to the exercise program or changes in $\mathrm{VO}_{2}$ peak by multivariate analysis. Finally, although there was no significant change during the 6 months of aerobic exercise in the subjects' ability to perform any of the 29 individual ADLs and IADLs described in the baseline and follow-up surveys, there was a trend for improvement in overall functional status ( $P=.08$ for all variables combined).

\section{DISCUSSION}

The results of this investigation indicate that 6 months of moderate-intensity, low-frequency aerobic training results in an increase in cardiorespiratory capacity in subjects aged 80 and older. Six months of aerobic training increased maximal ETT duration by $33 \%, \mathrm{VO}_{2}$ peak by $6.5 \%$, and maximal $\mathrm{O}_{2}$ pulse by $8.6 \%$. In addition, after training, mean HR was significantly lower during submaximal ETT stages, and resting systolic blood pressure was significantly lower. Using multiple regression analysis, baseline $\mathrm{VO}_{2}$ peak and the total time spent performing aerobic exercise training were independent predictors of the training-related improvements in $\mathrm{VO}_{2}$ peak.

The low mean frequency and duration of exercise achieved by our patients (two 20-minute sessions per week at $75 \%$ of maximal HR) can explain the smaller improvement in $\mathrm{VO}_{2}$ peak noted with training in the present study compared with prior exercise trials involving healthier subjects in their 60s and 70s. Seals et al. demonstrated that men and women aged $63 \pm 2$ increased their absolute $\mathrm{VO}_{2}$ peak by $10 \%$ after 6 months of 30 -minute aerobic training sessions 4.6 times per week at approximately

\begin{tabular}{llccr}
\hline \multicolumn{5}{l}{ Table 3. Multivariate Predictors of Training-Induced Changes in Maximal Oxygen Consumption $\left(\mathrm{VO}_{2}\right.$ peak $)$} \\
\hline Step & \multicolumn{1}{c}{ Variable } & $\beta$ & $\mathrm{r}$ & P-value \\
\hline Model 1 & & & \\
1 & & -0.35 & 0.75 & .002 \\
2 & Baseline $\mathrm{VO}_{2}$ peak, $\mathrm{mL} / \mathrm{kg} / \mathrm{min}$ & 0.55 & .008 \\
Model 2 & Total minutes of aerobic training & 0.003 & 0.718 & .005 \\
1 & Baseline $\mathrm{VO}_{2}$ peak, $\mathrm{mL} / \mathrm{kg} / \mathrm{min}$ & -0.344 & .012 \\
2 & Total bike minutes & 0.005 & 0.521 & .098 \\
3 & Total treadmill minutes & 0.002 & 0.365 & \\
\hline
\end{tabular}


$70 \%$ of maximal HR. ${ }^{13}$ Posner et al. similarly showed that three 40-minute training sessions at $70 \%$ of maximal HR resulted in an $8.5 \%$ increase in $\mathrm{VO}_{2}$ peak in subjects with a mean age of 69 years. ${ }^{11}$ Additionally, a meta-analysis completed by Green et al. of 955 subjects from 29 studies with 30-minute training sessions averaging three times per week at an unquantified intensity demonstrated that older individuals (mean age $=68$ ) increased their $\mathrm{VO}_{2}$ peak by $3.5 \mathrm{~mL} / \mathrm{kg} / \mathrm{min}$, or $14 \%$ on average over 6 months. ${ }^{17}$ In comparison, this study incorporated a significantly older and less-healthy sample who exercised at a lower frequency and session duration, resulting in an improvement in $\mathrm{VO}_{2}$ peak of approximately $50 \%$ to $75 \%$ of those seen in the above investigations.

Although the low frequency and duration of exercise sessions attained in this study probably limited the improvement in $\mathrm{VO}_{2}$ peak achieved, these same features may also have reduced the risk of orthopedic injuries and pain related to arthritis in this older population. ${ }^{21}$ It is noteworthy that no subjects dropped out of the present investigation due to injury, nor did any report an injury that limited exercise performance. The frequency and duration of the exercise sessions achieved in this investigation represent a training regimen that can be maintained by frail people aged 80 and older. The current findings suggest that such a program could attenuate the functional decline which often accompanies aging and sedentary behavior.

After 6 months of aerobic training, our questionnaire data showed a trend toward increasing ease in our subjects' ability to perform ADLs and IADLs $(P=.08)$. Morey et al. have demonstrated that fitness (morphological factors, muscular performance, and motor ability) are directly associated with functional status. ${ }^{10}$ The loss of the ability to perform ADLs and IADLs is a marker for impending disability in this age group. Regular physical activity and fitness have been shown to be associated with outcomes that can attenuate the onset of disability. ${ }^{10}$

These results demonstrate that the two most important independent predictors of an improvement in $\mathrm{VO}_{2}$ peak in subjects aged 80 and older are the pretraining $\mathrm{VO}_{2}$ peak and the amount of time spent performing aerobic exercise. Subjects with lower baseline fitness measurements experienced a greater increase in $\mathrm{VO}_{2}$ peak than subjects with higher values. This suggests that even deconditioned people aged 80 and older may improve their cardiorespiratory fitness with exercise training. Although time spent training on a stationary cycle and on the treadmill was similar $(9.3 \pm 2.9$ and $11.4 \pm 4.0$ minutes per session, respectively), time spent on the cycle was found to be a more important predictor of change in $\mathrm{VO}_{2}$ peak than that performing treadmill exercise. The stationary cycle may also provide a greater degree of stability and perhaps less risk of injury than treadmill exercise in this frail age group.

The focus of this study was to assess the feasibility of aerobic exercise training in relatively frail older subjects in a retirement community setting. The primary limitation of this pilot study is that subjects were self-referred and were not randomized to exercise or a nonexercising control group. It is therefore possible that unmonitored factors such as diet or nonaerobic physical activity contributed to the ob- served increase in aerobic capacity. Another limitation of our investigation is a lack of cardiac output measurements to investigate the mechanism for the improved aerobic capacity. However, the unchanged peak HR and increased $\mathrm{O}_{2}$ pulse after training are typical of findings in younger subjects and suggest a similar combination of central and peripheral adaptations to training. A further limitation is our reliance on questionnaire data to assess changes in ADLs and IADLs. Direct assessments of functional status changes with training in very old people should be addressed in future studies.

In conclusion, subjects aged 80 and older can achieve significant improvement in aerobic capacity from moderate-intensity, low-frequency exercise training. If applied on a large scale, such exercise training has the potential to reduce or postpone the high incidences of disability and improve the quality of life in this, the most rapidly increasing, segment of our population.

\section{REFERENCES}

1. Hagberg JM. Effect of training on the decline of $\mathrm{VO}_{2} \max$ with aging. Fed Proc 1987;46:1830-1837.

2. Buskirk ER, Hodgson JL. Age and aerobic power: The rate of change in men and women. Fed Proc 1987;46:1824-1829.

3. Fleg JL, Lakatta EG. Role of muscle loss in age-associated reduction in $\mathrm{VO}_{2}$ max. J Appl Physiol 1988;65:1147-1151.

4. Dehn ME, Bruce RA. Longitudinal variations in maximal oxygen intake with age and activity. J Appl Physiol 1972;33:805-807.

5. Tseng B, Marsh DR, Hamilton MT et al. Strength and aerobic training attenuate muscle wasting and improve resistance to the development of disability with aging. J Gerontol A Biol Sci Med Sci 1995;50A:M113-M119.

6. Buchner DM, Beresford SAA, Larson EB et al. Effects of physical activity on health status in older adults. II. Intervention studies. Annu Rev Public Health 1992;13:469-488.

7. Shephard RJ. The scientific basis of exercise prescription for the very old. J Am Geriatr Soc 1990;38:62-70.

8. Mazzeo RS, Cavanaugh P, Evans P et al. Exercise and physical activity for older adults. Med Sci Sports Exerc 1998;30:992-1008.

9. Shephard RJ. Extending independence in older adults. Geriatrics 1993;48:61-64.

10. Morey MC, Pieper CF, Cornoni-Huntley J. Physical fitness and functional limitations in community-dwelling older adults. Med Sci Sports Exerc 1998;30: 715-723.

11. Posner JD, Gorman KM, Windsor-Landsberg L et al. Low to moderate intensity endurance training in healthy older adults: Physiological responses after 4 months. J Am Geriatr Soc 1992;40:1-7.

12. Saltin B, Rowell LB. Functional adaptations to physical activity and inactivity. Fed Proc 1980;39:1506-1513.

13. Seals DR, Hagberg JM, Hurley BF et al. Endurance training in older men and women. I. Cardiovascular responses to exercise. J Appl Physiol 1984;57:1024-1029.

14. Blumenthal JA, Emery DF, Madden DJ et al. Cardiovascular and behavioral effects of aerobic exercise training in healthy older men and women. J Gerontol 1989;44:M147-M157.

15. Cunningham DA, Rechnitzer PA, Howard JH et al. Exercise training of men at retirement: A clinical trial. J Gerontol 1987;42:17-23.

16. Foster VL, Jume GJ, Nyrnes WC et al. Endurance training for elderly women: Moderate vs low intensity. J Gerontol 1989;44:M184-M188.

17. Kohrt WM, Malley MT, Coggan AR et al. Effects of gender, age, and fitness level on response of $\mathrm{VO}_{2}$ max to training in 60-71 years old. J Appl Physiol 1991;71:2004-2011.

18. Hagberg JM, Graves JE, Limacher M et al. Cardiovascular responses of 70to 79-year-old men and women to exercise training. J Appl Physiol 1989;66: 2589-2594.

19. Green JS, Crouse SF. The effects of endurance training on functional capacity in the elderly: A meta-analysis. Med Sci Sports Exerc 1995;27:920-926.

20. Cobbs EL, Duthie EH, Murphy JB. Geriatrics Review Syllabus, 4th Ed. New York: Kendall/Hunt, 1999, pp 347-348.

21. Williamson JD, Fried LP. Characterization of older adults who attribute functional decrements to 'old age.' J Am Geriatr Soc 1996;44:1429-1434.

22. Rooney EM. Exercise for older patients, why it's worth your effort. Geriatrics 1993;48:68-77. 